\title{
UnA CUESTIÓn DE Clase. Jordi, ELITISMO Y MARICONCRACIA EN "El DÍA QUE MURIÓ MARILYN"
}

\author{
Sergio Martínez Rey \\ Universidad de Utah
}

Title: A question of class. Jordi, elitism and "mariconcracia" in El día que murió Marilyn

\begin{abstract}
El día que murió Marilyn (1998) by Terenci Moix, in its capacity as a choral account of late-Francoist bourgeois society in Barcelona, might be one of the most outstanding examples of Spanish novel with intergenerational descriptive aspirations. The set of its narrator-characters build a complete picture of their era, contributing their perspective and locus of enunciation, deeply influenced by their sociohistorical context. However, perhaps the most exceptional figure among all of them is Jordi Llovet, Bruno's best friend and openly homosexual. Based on a detailed examination of this character's description and behavior in the novel, this study will aim to analyze the construction of a very particular notion of çlass"derived from the accumulation and leveling of different cultural references, and justified by the tension between elite and mediocrity, permanent in Jordi's perspective of the world. To this end, aspects related to this character's class consciousness, sexual identity and his immediate context will be taken into consideration, applying frameworks linked to queer studies, as well as relevant reflections regarding Moix's authorial trajectory.
\end{abstract}

Key words: Elitism. Mariconcracia. Camp. Queer. Late-Francoism. Homophobia

El día que murió Marilyn constituye uno de los más significativos retratos de la memoria sentimental tardo-franquista española, y más concretamente barcelonesa, en la novelística hispánica. Novela escrita por Terenci Moix, inicialmente publicada en catalán en 1969, su versión definitiva y revisada aparece finalmente en 1998 en castellano. En un ejercicio de narración polifónica a través de la memoria de sus personajes, la obra presenta los conflictos, tensiones e inconsistencias fundamentales de una generación educada en la rectitud moral católica-nacionalista pero testigo del preludio de la ruptura ideológica del régimen. A partir del relato de la vida de sus protagonistas, Moix construye un particular Bildungsroman cimentado en las visiones individuales de los miembros de dos familias burguesas que, en su lejanía enunciativa, completan una representación coral de la capital catalana desde los años cuarenta (Morales Guevara 2008: 172).

El presente estudio, sin embargo, se centrará en uno de los cinco personajes-narradores: Jordi, mejor amigo de Bruno, vástago de los Llovet 
y abiertamente homosexual. A partir de su narración, reflexiones, disquisiciones, y caracterización externa, se analizará la construcción de una noción muy particular de "clase" derivada de la acumulación y nivelación de diversos referentes culturales de la época. Esta clasificación sistemática se mostrará central en la descripción de las tensiones entre individualismo y comunidad, entre élite y mediocridad, definitorias en la evolución del personaje. Asimismo, por su relevancia y productividad analítica, este examen se llevará a cabo atendiendo a varias nociones ligadas a la teoría e historiografía queer tales como camp, urbanidad gay, cosmopolitismo, pecado, y el desdoblamiento existente entre cultura global, nacional y local.

En primer lugar, resulta conveniente destacar algunas claves generacionales en torno a la construcción del sujeto homosexual en la Barcelona a partir de los años cincuenta. Algunos de los hitos históricos más remarcables son la inclusión de homosexualidad en la Ley de Vagos y Maleantes en 1954, su posterior gestión a través de la Ley de Peligrosidad y Rehabilitación Social en 1970, y la primera manifestación multitudinaria por los derechos LGBT en 1977 en Barcelona, convocada por el Front dÁlliberament Gai de Catalunya (FAGC). Así, en líneas generales, el siglo XX del colectivo en España viene marcado por un proceso de criminalización de tendencias sexuales no heteronormativas, un ulterior viraje hacia la patologización y, finalmente, la consecución de la liberación, que culmina en la construcción de una comunidad, en la visibilidad colectiva frente a la represión individual.

No obstante, es necesario apuntar a la excepcionalidad de localidades como Sitges o Barcelona en la desenvoltura sociohistórica del sujeto homosexual (Olmeda 2007: 27). Esta diferencia esencial con respecto a otros lugares en el territorio nacional se justifica por tres razones: la preminencia del turismo, que implicó el advenimiento de influencias extranjeras; la mayor industrialización y riqueza de la región, que permitió la configuración de un núcleo burgués fuerte; y la naturaleza urbana del espacio, en contraste 
con una España mayoritariamente rural. En este respecto, Geoffroy Huard llegará a afirmar que en el periodo entre 1956 y 1980 “en Barcelona se desarolló una vida homosexual de alguna manera tolerada y visible para las autoridades" (2016: 150). Sin embargo, el académico también observa que, en base a los registros de la época, las acciones institucionales que se tomaron de forma efectiva lo fueron exclusivamente contra aquellos individuos que formaban parte de las clases populares. En cambio, en el corpus que maneja Huard, no existe referencia alguna a homosexuales de clase media o acomodada condenados por las autoridades a razón de comportamientos “invertidos". Para el autor, esta circunstancia evidencia una mayor visibilidad de la esperada del colectivo barcelonés, previa incluso a los movimientos sociales iniciados en los 70 (2016: 151).

Es en este contexto en el que Jordi se cría, desarrolla su personalidad y construye su identidad. $Y$ es precisamente este proceso de construcción identitaria el núcleo de nuestro estudio, al tiempo que constituye uno de los aspectos más destacados de la novela de Moix. En palabras de Jorge Marí, refiriéndose al ensamblaje del conjunto de los personajes-narradores en la obra: "el cine norteamericano mediatiza las percepciones de Bruno y otros personajes, guía sus deseos y su imaginación, les ofrece símbolos y metáforas que condensan su memoria y organizan sus discursos narrativos. El cine de Hollywood les aporta estrategias de supervivencia y mecanismos contra la soledad (2000: 245)". De este modo, como en buena parte de la literatura posmoderna, se presenta en la novela una serie de personajes cuya temporalidad y acceso a la realidad y a la memoria queden indefectiblemente marcados por una serie de hitos culturales contextuales.

En este sentido, de acuerdo con la tesis de Marí, se podría afirmar que Jordi construye su identidad a través de productos y moldes cinematográficos, a los que se han de añadir aquellos de naturaleza musical o literaria. Así, el personaje-narrador se construye a sí mismo a través proceso mimético de 
recepción artística. Sin embargo, el caso de Jordi reviste de una complejidad añadida debida a su condición homosexual y a su particular nivel socioeconómico. Por tanto, a esta mímesis primaria es necesario incorporar un proceso de selección, clasificación y jerarquización de referentes, que deriva en una construcción muy particular del "otro". Esta faceta complementaria se intuye muy tempranamente en la novela:

De June Allyson, pecosa como yo, pero no tan rubia, imitaba aquel su aire deportivo, moderno, dinámico sin caer en la ordinariez. De Amèlia Quadreny ambicionaba aquella imperiosidad de cada gesto, aquel ritmo grandioso, frívolo y sereno a la vez, que la hacía triunfar sobre cualquier circunstancia: que la divinizaba. De Andreu aspiraba a captar aquella elegancia imposible de ser descrita, propia de los seres que se han autosublimado dejando atrás la vulgaridad en que quería encerrarlos su circunstancia, por demás mediocre. (Moix 1998: 151)

Resulta imposible obviar en este fragmento una oposición semántica central entre conceptos tales como "moderno", "grandioso” o "elegancia”, frente a "ordinariez", "vulgaridad" o "mediocre". Radica en esta contraposición la base de las aspiraciones identitarias de Jordi, su locus de enunciación en la historia, su trayecto hacia la madurez. Por otro lado, este contraste entre el sujeto inicialmente imitado y aquel asociado al contraejemplo, la otredad, se ve influido por una serie de factores que confluyen en una jerarquía asociada a códigos culturales internos del colectivo homosexual, así como a las nociones de burguesía y popularidad.

En un ámbito puramente socioeconómico, la autoconciencia de clase aparece a lo largo del relato de Jordi de forma explícita y reiterada. En este sentido, podría decirse que existe una primera oposición ante "el otro" derivada de la identidad burguesa del personaje-narrador. Esta se materializa principalmente en las inquietudes personales de Jordi, tales como su viajehuida a París o la perspectiva de heredar el imperio editorial de su padre. Sin embargo, es también posible observar esta oposición identitaria en su descripción de las clases obreras barcelonesas: 
En las dunas bailaban gitanillos medio desnudos: cuerpos ennegrecidos, costras de mocos y durezas, pies descalzos con otra costra de fango y suciedad. Y yo contemplaba mi jersey de moda, muy grueso, con soberbios rombos blancos sobre un fondo de lana verde, y sentía un orgullo que poco a poco me hacía considerarme superior al resto del mundo. [...] Era aterrador pensar que algún día podíamos encontrarnos así. Después, siempre que he intentado evocar a aquella gente, nunca lo he logrado del todo. Sus miradas pertenecían a una especie de raza irreal que estaba muy lejos de la nuestra; sus hechos diferenciales estaban más allá de cualquier posibilidad de aproximación que pudiéramos realizar nosotros: nunca existieron o tal vez no los vi. (Moix 1998: 156)

Esta contraposición resulta ciertamente radical, al punto de que las clases bajas quedan definidas como "raza irreal” sistemáticamente invisible para el narrador. Del mismo modo, Jordi elabora un discurso dual entre el “yo" y un "nosotros", entre sí mismo y la colectividad. Por un lado, Jordi reafirma su conciencia de clase en la expresión de su miedo a la pobreza, así como en el uso del término "raza" percibido como "nuestra". Por otro, resulta muy destacable la manifestación de un orgullo asociado a un juicio de sí mismo como "superior al resto del mundo", que de acuerdo con el propio Jordi se ha ido formando "poco a poco", progresivamente.

Esta tensión niveladora entre entorno e individuo es nuclear en la caracterización de Jordi. De hecho, retomando su idea de raza burguesa, se presentará a lo largo de la obra una paulatina separación entre sí mismo y el mundo burgués urbano, paralela a su proceso de construcción identitaria. Así, consideramos que este distanciamiento es explicable a través de su condición de homosexual y, muy particularmente, de su distintiva concepción de la cultura. Jordi encuentra sentido a su realidad a través del cine y la literatura y maneja su propio canon, basado en asociaciones entre el sujeto y ciertos moldes extraídos de la cultura popular, de masas y global. No obstante, este canon va mutando y en cierto momento, Jordi manifiesta su desinterés por la cultura burguesa. A partir de este punto, el joven se ve a sí mismo envuelto en preocupaciones de calado más artístico, inmaterial, y a su juicio, más elevadas: 
Y es que mamá había sabido recoger aquel caudal de conocimientos varios y dispersos — de todo un poco, lo llamaban-, la sabiduría barata y de circunstancias, que durante mucho tiempo ha sido la herencia cultural de muchas burguesitas del Ensanche. Revivo el piso de los abuelos como el ejemplo más puro, extremadamente conmovedor y nada aislado, de este patrimonio que tenía como ideal de un non plus ultra cultural las veladas operísticas escuchadas en el tercer piso del Liceo, que si Andreu era la personificación del analfabetismo de cierta clase media, Benlloc representaba toda la estafa cultural de la burguesía. (Moix 1998: 146)

Jordi contrapone de nuevo dos realidades, la burguesía catalana y una suerte de aristocracia cultural, ubicándose en todo momento en la facción superior, o al menos aspirando a ella. En este sentido, su relación con Andreu, e incluso con Bruno, y los cambios que estas experimentan en el proceso de madurez de Jordi, marcan de manera muy clara este cambio de perspectiva desde la mímesis indiscriminada al endurecimiento de los criterios de evaluación de los individuos y los referentes culturales que le rodean:

(Yo no comprendía que te empeñaras en buscar una cultura al alcance de todo el mundo, ya que eso no podía encontrarse de ninguna manera con mi idea - iy que me dure! - de la selectividad del espíritu artístico.) La tarea didáctica de Andreu, tarea por otra parte primaria, terminó precisamente en el momento en el que más me necesitaba, pero en el que ya no podía seguirme: aquel momento en que su propia obra -es decir, Yo- tenía aspiraciones algo más elevadas que su repertorio «imprescindible» para brillar en sociedad. (Moix 1998: 133)

En cierto punto de su desarrollo, Jordi evidencia una desatención absoluta hacia su papel en la sociedad y una autoconciencia de carácter profundamente elitista. Así, su pertenencia a una supuesta aristocracia cultural elevada representa un modo de identificación radical con el margen, que no se localiza tanto en la incapacidad de integración, como en la superioridad frente a la norma o lo esperado. Esta postura es explícita incluso en su descripción de su despertar sexual, que narra sin asomo de sentimentalismo o emotividad. A pesar de tratarse esta de una experiencia de desarraigo traumática, Jordi la racionaliza en sus escritos, y la percibe como parte de una serie de procesos que le conducen a su personal conciencia de superioridad: 
Por el contrario: desde un principio tuve la impresión de pertenecer a una especie de aristocracia, un grupo privilegiado que estaba muy por encima de vosotros, tan descuidados, vulgares y groseros en gestos y palabras. [... Acabábamos de darnos cuenta de que la sociedad tenía dictadas una serie de leyes que me apartaban de su seno, que me rechazaban para recluirme definitivamente en el grupo de los Andreu «la Medallona.» [...] Entonces creía aún que el futuro más brillante sólo se podía conseguir perteneciendo al grupo de las maricas selectas, y hasta me consideraba con derecho a despreciaros por ser demasiado normales. [...] Mi evolución desde el dolor hasta el cinismo me inmunizaba contra los ataques de esas personas increíblemente felices que un día se casan y tienen hijos que esperan con ilusión los juguetes de los Reyes. (Moix 1998: 134-135)

De nuevo, Jordi maneja en este fragmento confesional unos criterios jerarquizadores que oscilan entre la vulgaridad o la ordinariez, representadas en este caso por sus compañeros heterosexuales, y un grupo selecto, las maricas. Cabe notar además que, al igual que en su mímesis fílmica, esta clasificación no obedece simplemente a una diferenciación en términos de sexualidad o comunidad, sino que posee un calado profundamente estético, condicionado por "gestos y palabras". Es en este punto en que la noción de camp se hace imprescindible en la caracterización de Jordi, especialmente si se contempla en el contexto de la producción novelística de Terenci Moix.

En su artículo "The Subversive Processes of Camp Production in the Novels of Terenci Moix", Timothy McGovern describe el mundo camp del autor catalán como un universo narratológico en el que el paradigma heteropatriarcal se ve desvirtuado a través del humor, y en el que los hombres heterosexuales, sexistas y homófobos ocupan las posiciones más bajas de la escala social, siendo objetos de los antojos de los personajes no normativos (2004: 45). De acuerdo a este autor, esta superioridad cualitativa se asocia o justifica a través de una supuesta especial cognición queer, una visión que alcanza mayor complejidad y amplitud que la heteronormativa, semejante a la "sensibilidad gay" acuñada por Susan Sontag.

No obstante, cabe mencionar que el acercamiento de McGovern a la obra de Moix se enfoca principalmente en dos novelas, Garras de astracán 
y Mujercísimas, de planteamiento bien distinto a El día que murió Marilyn. Mientras que las primeras son ficciones construidas a partir de personajes y eventos profundamente ligados al camp desde un inicio, casi paródicos, con un fuerte componente irónico, aparatoso y ciertamente absurdo, la obra que nos atañe, por su naturaleza de novela de aprendizaje y retrato generacional, no resulta tan marcadamente humorística. En su lugar, en El día que murió Marilyn la subversión del sistema heteropatriarcal tiene lugar a través de un desvelamiento de sus mecanismos de acción a partir las experiencias y pensamientos de los personajes en su proceso hacia la madurez, tal y como se evidencia en el fragmento anterior.

En esta misma línea, el teórico Alexander Doty define el camp como: "an attitude at once casual and severe, affectionate and ironic, which served to deflate the pretensions of mainstream culture while elevating what that same culture devalued or repressed" (2012: 11). Así, siguiendo esta definición, el camp habilitaría al sujeto queer para la reescritura de las representaciones oficiales de la cultura de masas al tiempo que desvelaría la sensibilidad gay presente en todo producto de la cultura popular. Sin embargo, el autor establece un hito sociohistórico que marca un antes y un después en la recepción queer, un viraje en las implicaciones del concepto de camp: los disturbios de Stonewall en 1969. Doty defiende la existencia de una lectura gay pre-Stonewall, marcada por la identificación e iconización de sujetos femeninos, asociada a una absorción extravagante de las estrellas. Sin embargo, tras los disturbios este estado primario se vería sustituido por una recepción menos basado en lo estético: "the natural-man discourse, with its strong political and social vision and its sense of a fulfilled and open self" (Doty 2012: 75). En fin, esta fluctuación resultaría en un viraje hacia la militancia, desembocando en la construcción de un sujeto político colectivo con agencia y que demanda cambios en el sistema heteronormativo. 
A priori, se ha de hacer notar la esencial historicidad y localismo de este acercamiento. El autor se refiere exclusivamente a la comunidad queer en Estados Unidos y a un evento que propulsó la visibilidad del colectivo y lo acercó a nuevas formas de activismo. En este sentido, equiparar este proceso al caso español o barcelonés resulta como mínimo problemático. No obstante, paralelamente, hemos de resaltar la alineación de ciertos aspectos o factores significativos: la importancia del cine americano en la construcción de los personajes de la novela, el mayor aperturismo de Barcelona como centro urbano cosmopolita, y la naturalmente asumida equivalencia entre Stonewall y las manifestaciones convocadas por la FAGC en 1977.

Considerando todo lo anterior, parece claro que el componente camp de la recepción de Jordi se corresponde mayormente a una concepción típicamente pre-Stonewall, basada en la mímesis fascinada, la iconización y la aplicación de moldes cinematográficos sobre la realidad, con especial énfasis en modelos femeninos tales como Marilyn Monroe o la altanera y divina Amèlia. Se da en el pensamiento del personaje, por tanto, una diferenciación identitaria fundamentalmente estética que, si bien marginal e inherentemente anti normativa, conlleva unas motivaciones de menor calado social y comunitario, al menos en lo explícito. Cabe recordar que la redacción de esta novela en lengua catalana se inicia en los años 60 , en los cuales una lectura pos-Stonewall del sujeto homosexual resulta ciertamente imposible.

Pareciera que la diáfana tendencia al elitismo por parte de Jordi impide su identificación con cualquier colectivo. En su proceso hacia la madurez, se produce una evidente falta de afinidad con ningún grupo familiar, nacional, local, ni siquiera sexual. Esta actitud, no obstante, no resulta extraña en el contexto de la denominada literatura "pre-Stonewall”, en la que una de las líneas o topos fundamentales es la consideración del sujeto gay como reconocidamente exótico o marginal, inherentemente renegado y conscientemente transgresor del sistema (Brookes 2009: 60). Si bien hemos apuntado 
a la existencia de una conciencia de clase burguesa, esta es muy parcial. Jordi solamente se sirve de ella para distanciarse del otro, de las clases bajas, típicamente asociadas a la miseria económica y cultural. En sus propias palabras, refiriéndose a la clase obrera: "en conjunto, como clase social o como individuos, no aportan lo que se dice ni así de comunicación estética. Y es bien sabido que todo lo que no sea estética no es nada” (Moix 1998: 157).

No obstante, en realidad, tal y como ya se ha indicado, Jordi termina por despreciar también su propia clase, y ansía alejarse de toda expectativa asociada a su pertenencia a este colectivo, en especial en lo que se refiere a su faceta de heredero del negocio de los Llovet. No es casual que Jordi identifique la editorial dirigida por su padre con la frivolidad y superficialidad burguesa, afirmando: "La Editorial Llovet fue subiendo fuerte y poderosa, siempre a partir de una demagogia que excluía la menor posibilidad de seriedad intelectual y se acogía a las ventajas que ofrecía el gusto de una sociedad tremendamente embrutecida" (Moix 1998: 148). Su manifiesto desinterés por el aspecto económico y su afán estético, resultante en su inclinación al arte "superior", conducen a Jordi al desarraigo respecto a la realidad burguesa.

Otro de los aspectos fundamentales de la reticencia de Jordi hacia la clase burguesa es su papel histórico en la normativización franquista. En este sentido, en el plano de lo esencialmente nacional, Jordi parece asumir que la sombra del franquismo cubre indefectiblemente todo lo hispánico. Su consideración de la cultura nacional se identifica con su educación, religiosa y moralista, que bebe de los grandes relatos y mitos de la tradición nacionalcatolicista. El mismo Andreu se percata de esta filiación franquista-nacional por parte del joven y trata de contradecirle: "Y no te armes un lío, ahora, con el concepto de patria que os enseñan en el colegio, que es totalmente distinto. No me refiero a amores de guerra novelesca, de defender el país 
hasta la muerte y dejarte matar y todo eso... No, no te pido que seas un héroe. Sólo me refiero a tus raíces" (Moix 1998: 158).

Ante el espíritu supremacista e individualista de Jordi, es precisamente Andreu el personaje que más frecuentemente trata de contradecirle y forzarle a enfrentarse a una realidad externa que no se corresponde con sus expectativas y aspiraciones aristocráticas:

-iDiferente! ¡Que te crees tú eso, majo! Es la mejor excusa que tendrás para luchar contra la vergüenza: procurar convencerte a ti mismo de que, en el fondo, eres diferente de estas mariconas que vienen a hacer el poseur dos o tres veces por semana, saliendo de la oficina. Por lo visto, en eso como en todo, hay clases: también existe una aristocracia en nuestra sociedad: maricas más elegantes, más cultas, más serias que las demás. Pero hay un momento en que todas somos iguales, aunque te duela: la misma ralea, la misma necesidad de placer cueste lo que cueste. Todas como perras solitarias que se buscan por los bares oscuros o por los cines de barrio. Los hay que intentan sublimarlo, pero, a fin de cuentas, la esencia es la misma... (Moix 1998: 162)

Y en este afán de diferencia que denuncia Andreu radican las tensiones existentes en la interseccionalidad identitaria de Jordi. Toda consideración o categorización colectiva, ya sea nacional, socioeconómica o política, está condenada a la otredad en la personal construcción de la interioridad del personaje. No obstante, quizá el aspecto de este consciente aislamiento supremacista que suscita mayor interés es aquel relativo a cuestiones más próximas a las problemáticas del sujeto queer.

Dentro del colectivo, y considerando la teorización anterior en su totalidad, es posible observar una suerte de jerarquización de maricas en el pensamiento de Jordi, que el propio Andreu reconoce. Así, del mismo modo que la construcción de las percepciones en los personajes está fuertemente condicionada por el cine (Marí 2000: 24), Jordi basa sus cribación en el valor cualitativo asignado a ciertos referentes culturales, aplicando este filtro en dos niveles: por un lado, definiendo a los sujetos homosexuales de acuerdo con el tipo de productos culturales que consumen; por el otro, identificando estos individuos con figuras de la cultura popular. 
En el primer caso, merece especial atención la consistente consideración negativa de artistas españoles masivamente populares durante los años 50 y 60, con particular desprecio a las cupletistas, folklóricas y copleras de la época:

Todo eso de llevarme a jugar a la canasta con lesbianas demimondaines, de presumir de nuestro asunto en los estrenos de las folklóricas que os gustaban, de exhibirme como un triunfo ante tus amigos ajados, de caras mugrientas bajo el cosmético. [...] Y tú, Andreu, tú igual que ellos: locas de libros carísimos que nunca abríais, de discos de Bach y Haydn que nunca podíais escuchar porque Sara Montiel ocupaba todo vuestro tiempo y el que os quedaba lo dedicabais a Luis Mariano... Y yo, Andreu, yo que soñaba con el infierno de Rimbaud y Verlaine como realización de un sentimiento edificado sobre el afán creador de almas verdaderamente selectas (Moix 1998: 161- 162)

La concepción de 'locas' construida por Jordi, por ende, queda indefectiblemente marcada, además de por ciertos rasgos físicos y conductuales, por aquello que escuchan, ven o leen, y aquello que no. Existe para Jordi una distancia abismal entre Sara Montiel, española, aparentemente afín al régimen, y popular en la nación, muy especialmente entre la comunidad homosexual (Álvarez Rodrigo 2019: 3), y artistas excelsos como Bach o Verlaine, extranjeros, fallecidos, cosmopolitas y marcas de intelectualidad selecta.

El interés de esta distinción cualitativa entre artistas autóctonos y foráneos radica en su configuración en múltiples niveles. En primer lugar, debemos destacar la asociación inevitable de personajes del cine nacional de la época, así como del género musical de la copla o la canción española, con el Régimen. Las autoridades franquistas auspiciaban a estas estrellas y condicionaban su interpretación, independientemente de la ideología o las implicaciones potencialmente subversivas de la vida y obra de estas divas. De hecho, uno de los principales cauces de representatividad de estas celebridades es su identificación con la nación española per se, la esencia vernácula 
del territorio, castizo y culturalmente unificado por la definición de España manejada por el poder (Fuente Cuesta: 58).

Simultáneamente, se ha de resaltar la inmensa popularidad de artistas como Sara Montiel, Concha Piquer o Lola Flores, todas ellas mencionadas en la novela de Moix. Estas estrellas eran tan reconocidas por el bando delos vencedores como por el de los vencidos tras la Guerra Civil, tanto por la burguesía como por las clases menos privilegiadas una vez el Régimen está asentado (Sieburth 2014: 5). Por el contrario, las obras de Verlaine o Rimbaud no estaban al alcance de todos, sino de una minoría intelectual que podía superar la censura sistemática, especialmente al inicio de la dictadura, leer en un idioma extranjero o hacerse con buenas traducciones. Considerando las tendencias elitistas de Jordi, no resulta descabellado pensar que esta oposición entre cultura de masas e intelectualidad selecta juega un papel crucial en su juicio artístico.

Finalmente, el éxito de estas celebridades entre la comunidad homosexual española desde una época muy temprana es ciertamente notoria. Alberto Mira destaca el papel de las grandes copleras y actrices durante el franquismo como objeto de imitación ultrafemenino e hipersexualizado para el colectivo gay español, comparándolas con iconos internacionales como Mae West o Marilyn Monroe (2007: 418). Estas artistas, en su calidad de estrellas para las masas, se convierten en catalizadores de una lectura puramente camp de las narrativas nacionales, de un modo muy similar a la recepción típicamente pre-Stonewall descrita por Doty (2012: 75). En este sentido, conviene apuntar al hecho de que la era de esplendor de estas divas como modelo de las travestis nacionales finaliza justamente con la transición a la democracia, la entrada del país a la posmodernidad (Mira 2007: 439), a una etapa pos-FAGC.

Sin embargo, estas artistas, sus filmes y su música, siguen en plena vigencia durante la juventud de Jordi. En la niñez del personaje, estas can- 
tantes se mantienen como referentes de las denominadas "españoladas", que Ortega define como "conjunto amalgamado de referentes iconográficos y temáticos, que contagian acciones y caracteres y se fijan y asocian a una cultura nacional [que] se presentaría como un espacio de construcción donde entra en juego el cruce de miradas, el reconocimiento de lo propio en la representación externa." (2012: 102). En otras palabras, este término hace referencia a una serie de manifestaciones culturales caracterizadas por un uso exagerado de tópicos, estereotipos y lugares comunes en torno a lo castizo, sean o no reinterpretados a través del camp. Todo ello resulta, mediante la particular clasificación cultural de Jordi, en una reacción cosmopolita de rechazo a las grandes de la canción española, por su condición de castizas, populares, y gays en simultaneidad.

Partiendo de estas consideraciones, Jordi aplica sus conocimientos culturales, a priori jerarquizados, para criticar mordazmente a las 'maricas' de su entorno, a través de su identificación con ciertos personajes, patrones o moldes cinematográficos o literarios:

Arturu, pequeño pseudodandy de salón de la Diagonal, con sabiduría menos que superficial de todas las cosas à la page, podría practicar muy a gusto su frivolidad de vestal cursi. Poseía, claro está, aquel don tan estimable para hacerse una reputación en el seno de esta Sodoma sainetesca. (Moix 1998: 121)

Ah, locas de playa, presumidos pájaros de paso que no pasabais de Oscar Wilde -y decíais Uaild, para que se viera que ibais a la Berlitz- o de las novelas y las obras de teatro que dedicasen un poco de atención a vuestro problema sexual; vosotros, que sólo pensabais en el placer y el lucimiento de vuestros cuerpos esperpénticos. (Moix 1998: 161)

En suma, Jordi condena a las "locas" de Barcelona, aquella "Sodoma sainetesca”, por su frivolidad y superficialidad. En este sentido, no debemos obviar el hecho de que precisamente son estos mismos rasgos por los que Jordi ataca a la burguesía, siendo ambas, su orientación sexual y su clase, facetas inseparables de su propia identidad. No obstante, existe una diferencia 
fundamental entre ambos componentes identitarios renegados, y esta se encuentra en el terreno de lo material, en la realización física de la sexualidad, asociada a la corrupción y al pecado. Así, Jordi terminará describiendo un "nosotros" como "criaturas irremediablemente condenadas a la corrupción" (Moix 1998: 119), al tiempo que se distancia del resto de la comunidad gay barcelonesa afirmando:

Claro que lo que ellos no podrían aceptar era el hecho de que en nuestro asunto, aparentemente vicioso, la pureza triunfaba sobre la corrupción; y al hablar de enemigos incluyo tanto a la gente normal como a las amistades de Andreu -capitaneados por Arturu «la Marlene» y Rafael, «la Suzie Wong». (Moix 1998: 132)

-Acepto mi corrupción, la asumo de veras, pero sólo mientras pueda encauzarla por el buen camino, a partir de mi voluntad de pureza. La acepto como una prueba muy dura que Dios me ha impuesto, pero nunca como una imperiosidad de placer. Si dentro de la corrupción es posible el orden, yo no dejaré que el desorden me destruya del todo. Quiero que lo sepas de una vez, Andreu: yo nunca seré como los demás. (Moix 1998: 162)

$\mathrm{Su}$ ostensible desprecio a los individuos que conforman el colectivo evidencia la existencia de cierto grado de homofobia internalizada en Jordi, que se manifiesta en su "voluntad de pureza". Se establece de nuevo una radical oposición entre sí mismo y el otro, superficial y resignado al pecado carnal, identificado con los maricas de su círculo social. Esta homofobia, por otro lado, se originaría necesariamente a partir de su educación en el régimen franquista en el contexto burgués catalán, realidades que Jordi simultáneamente rechaza en su búsqueda de excelsitud a través de un supuesto superior espíritu artístico. En este sentido, la literatura en torno a la homofobia internalizada, que defiende la existencia de una coacción sistemática por parte de estructuras religiosas y construcciones nacionales cimentadas en la masculinidad (Kubicek et al. 2009: 602), apoyaría esta lectura.

A este respecto, quizá resulte conveniente retomar el análisis comparativo de El día que murió Marilyn con la tradición literaria “pre-Stonewall”. 
En su artículo "Inmaculate Manhood” Harry Thomas defiende la existencia de un tipo particular de hombre homosexual que surge a mediados del siglo XX en el panorama literario americano: aquel que trata de reafirmar su masculinidad a través de la conservación de roles de género típicamente varoniles, que detesta profundamente al afeminado y lo considera parte de una categoría inferior (2013: 597). Si bien Jordi no da muestras de una visión tan marcadamente machista, su descripción elitista y ciertamente plumófoba del colectivo coincide con aquella llevada a cabo por Jim Willard, protagonista de The City and the Pillar de Gore Vidal, quien deshumaniza a los hombres de tendencias femeninas, reduciéndolos a "strange womanish creatures" (2003: 66).

No obstante, con esta tendencia en mente, es necesario volver a apuntar a la importancia nuclear de modelos de comportamiento positivos femeninos en el desarrollo de la personalidad de Jordi, que brillan por su ausencia en los personajes descritos por Thomas. Así, la homofobia internalizada de Jordi no parece vincularse tan claramente a una misoginia estructural patriarcal. En su lugar, se relaciona con una noción muy particular de "corrupción", ligada a las ideas de pecado y enfermedad, ambas vertientes históricas de la ideología franquista en el tratamiento legal de la homosexualidad.

En este punto de la caracterización de Jordi, el célebre ensayo de Alberto Mira De Sodoma a Chueca (2007) ofrece un marco más localizado en la historia cultural española que puede ser de utilidad para los propósitos de este estudio. Mira defiende la existencia de tres matrices discursivas diacrónicas en la representación del colectivo homosexual en la España del siglo XX. Por un lado, el modelo malditista se asocia al sujeto homosexual consciente de su patologización y antagonización sistemática y que tiende a la absorción de moldes decadentistas. El segundo eje responde a un esquema homófilo, aquel que se aferra a la posibilidad de integración del homosexual 
en la sociedad basada en su supuesta condición de ente normativo, al menos potencialmente. Finalmente, el acercamiento camp representa un cuestionamiento de la rebeldía y la asimilación manifestadas por las anteriores visiones a través de la ironía y el uso de imágenes de la cultura de masas.

Si bien estos modelos no son excluyentes, lo curioso del caso es que Mira coloca inequívocamente a Terenci Moix en el tercer grupo de autores, destacando su humor y su erudición en lo que respecta a la cultura popular nacional y global (2007: 339). Así, retomando el examen del elitismo de Jordi, nos encontramos con un problema similar al presente en estudio de McGovern. La generalidad de la obra de Moix invita ciertamente a una caracterización camp tendente a la utilización recursos paródicos, así como en la descripción de eventos y personajes estrafalarios, inspirados por la cultura pop. Sin embargo, en el relato de la evolución hacia la madurez de Jordi, al menos en la versión de 1998, el componente humorístico camp no es tan relevante como el ejercicio de análisis y jerarquización a través de referentes culturales.

El propio Mira destaca en otra ocasión el uso de la cursilería como medio de ridiculización de los homosexuales de clase media en la obra temprana de Moix. No obstante, el académico también apunta a una corrección posterior localizada en los años noventa, motivada por la epifanía de la "perspectiva gay" en el autor catalán, que se manifiesta en la eliminación parcial de las descripciones homofóbicas en sus personajes (Mira 2016: 197). Se ha de recordar, pues, en este punto que la primera versión de la novela en catalán se publica en una fecha tan temprana como en 1969, mientras que su última versión en español no sale a la luz hasta 1998. Si bien el mismo autor reconoció en su momento que la principal razón para la reescritura de sus obras fue la adaptación a un contexto sin censura institucional (El País, 1998), los cambios con respecto al reflejo de la comunidad gay son también sustanciales. 
Traspasando los límites de la novela objeto de este estudio, la obra autobiográfica del autor catalán apunta a una relación de correspondencia entre el relato de su propia trayectoria vital y el tratamiento del sujeto homosexual en su producción literaria. Al igual que Jordi, Moix parece en ocasiones debatirse entre un "yo individual" juvenil, víctima particular del entorno en su condición de queer, y un posterior "yo colectivo", un nosotros que "le excluye de la «normalidad» [y] le aboca a la perspectiva diversa y creativa de la cultura" (Fone, 2019: 191), al tiempo que "alude al colectivo que reivindica la bandera de la homosexualidad, faceta personal que constituye el rasgo diferencial del personaje y el componente más explotado y desarrollado en la escritura de la memoria personal" (Fone, 2019: 194). En base a nuestro análisis previo, resulta evidente que este desarrollo ideológico autoral impacta en la descripción del personaje homosexual en El día que murió Marilyn, especialmente si se considera su publicación inicial en 1969 y su reescritura posterior.

En cualquier caso, la asunción inherentemente homofóbica de sí mismo como corrupto y la tendencia elitista de Jordi neutralizan de alguna forma el cuestionamiento de los márgenes moralistas y dicotómicos de la sociedad propio del camp, que no entiende de normas sociales o límites en el buen gusto. En este sentido, aplicando el marco propuesto por Mira, resulta imposible obviar la carga malditista de Jordi que, si bien expresada por cauces formales asociados al camp, supone la preservación de las ideas de clases, (in)moralidad, y degeneración. Jordi es, por tanto, un personaje atrapado entre ambas perspectivas históricas, la decadente y la camp, lo que podría ser explicado atendiendo a la naturaleza de El día que murió Marilyn: una novela de crecimiento, coral, de calado intergeneracional, en la que las circunstancias sociohistóricas tardo-franquistas tienen una importancia capital, y enmarcada en la producción peculiar de Terenci Moix. 
A la luz de estas consideraciones, el sentimiento de pertenencia a una élite dentro de la clase social y de la comunidad sexual de Jordi, aun en su reconocida condición de corrupto, resulta más comprensible. En suma, su construcción identitaria se vincula a una aspiración a la diferencia, a la unicidad, a un desprecio por la otredad, incluso a razón de aquello que en principio definiría su propia identidad. Jordi, a través de una serie de mecanismos clasificadores basados en sus referentes culturales y una noción de moralidad ciertamente problemática y compleja, establece un modelo de aristocracia, o mejor mariconcracia, de la que se siente parte.

Un autor que maneja el concepto de “mariconcracia” es Frédéric Martel, quien en su ensayo Global Gay, define este término como "a derogatory term that describes the overly rapid upward mobility of gays" (2018: 23). Se ha de notar que, al igual que en el caso de Jordi, la mariconcracia descrita por Martel obedece a la creación de una élite dentro del colectivo. Sin embargo, existen dos diferencias fundamentales entre estas dos posturas: la primera es que el mencionado en Global Gay es un concepto de carácter despectivo, surgido de una conciencia de comunidad que critica a aquel individuo que cree formar parte de otra clase diferente a la de sus iguales; la segunda es que, mientras que Martel se refiere a una clasificación de naturaleza económica, basada en el poder adquisitivo y un mercado global, la mariconcracia de Jordi es esencialmente cultural. Así, es posible atribuir esta diversificación, al menos en parte, a un estadio pre-Stonewall, o pre-FAGC, o tardo-franquista, de la conciencia de comunidad de Jordi, para quien los sujetos gais no forman un colectivo frente a opresiones estructurales, sino que su condición se limita a la de "criaturas irremediablemente condenadas a la corrupción”.

Habiendo examinado las tensiones identitarias existentes en la construcción de Jordi en sus múltiples niveles, resulta claro que el personaje ansía distanciarse de su entorno a través de la configuración de un elitismo 
o clasismo de carácter mayoritariamente cultural. Jordi maneja su propio canon y asigna diferentes valores a diversos productos culturales al tiempo que los asocia a los individuos de su círculo social. En este proceso, el joven se localiza, o pretende localizarse, consistentemente en la clase superior, despreciando al otro. Sin embargo, esta otredad no se identifica simplemente por las referencias culturales que manejan sus miembros, sino que tiene un calado socioeconómico, geográfico e incluso sexual muy destacado. De este modo, los artistas vinculados a la burguesía y a la comunidad gay local quedarán definidos por su frivolidad y simpleza, muy especialmente si son españoles.

En su proceso hacia la madurez y el descubrimiento del mundo y de sí mismo, Jordi comienza por una búsqueda desesperada de moldes que encajen con su sensibilidad temprana, asociados a la cultura cinematográfica de la época y la estética camp. No obstante, a partir de su adolescencia Jordi termina creando una personal taxonomía cualitativa de su realidad, identificando al otro como ser inferior. En su cruzada contra la mediocridad, contra todo lo que le es ajeno, incluye aquello que a priori le caracterizaría de un modo superficial como burgués, español, barcelonés y homosexual. Para Juan Bonilla es este precisamente el impulso de la generación representada en la novela, una huida de la mediocridad de los padres, de su cobardía y sus herencias, que desemboca en la construcción de un "inmenso caparazón de ficciones" que capacita al personaje para liberarse del lastre de un pasado que no es el suyo (Bonilla 2011: 39). Así, en la mirada crítica de Jordi, la comunidad gay barcelonesa se vería reducida a locas y maricas pecaminosas y vanidosas, mientras que la burguesía local se limitaría a un grupo de ricos embrutecidos y simplones.

No obstante, como colofón a este estudio, conviene ahondar en las posibles motivaciones de este particular elitismo. Para ello, se ha de apuntar al sistemático aislamiento al que Jordi se ve sometido desde su infancia. 
En este sentido, la construcción de sí mismo a través de patrones fílmicos y su configuración de una ideal aristocracia cultural no dejan de ser indicios de una concepción de sí mismo como diferente al resto, como queer. Jordi se fuerza a justificar su soledad por medio de su afán de superioridad, de su profundo individualismo, como modo de autodefensa ante los ataques estructurales desde todos los frentes: nacional-catolicista, familiar y comunitario. Así, su lectura camp de productos cinematográficos y su consiguiente aplicación a la realidad, esencialmente heteronormativa, apoyan también esta visión.

En última instancia, Jordi no es capaz de liberarse de su propia homofobia internalizada, que aparentemente no puede encontrar salida en ausencia de un sentimiento de pertenencia a un colectivo, en su predisposición al individualismo. Lo que en un principio podría interpretarse como una caracterización presuntuosa, una opinión de sí mismo que roza el delirio de grandeza, se debe realmente a un profundo desarraigo, producto de una exploración atormentada de su propia identidad no deseada. No podemos olvidar que esta construcción elitista, el establecimiento de la mariconcracia cultural en el pensamiento de Jordi, es esencialmente aspiracional, no efectiva, y, por tanto, en sus esperanzas de pureza, de mejora, existe una conciencia clara de sí mismo como ser imperfecto.

BiBliogRAFíA

versalÁlvarez Rodrigo, Álvaro (2019), Sara Montiel: Las Transgresiones al Ideal de Genero Franquista en El Ultimo Cuple (1957), Origen de un Icono Gay de la Democracia, Biblioteca Virtual Miguel de Cervantes. URI: http://hdl.handle.net/10045/96030

Bonilla, Juan (2011), "En pos de Terenci Moix”, Cuadernos Hispanoamericanos, N. ${ }^{0}$ 728, pp. 31-39. URL: http://www.cervantesvirtual.com/obra/en-pos-de-terenci-moix/

Brookes, Les (2009), Gay Male Fiction Since Stonewall: Ideology, Conflict, and Aesthetics, New York: Routledge.

Doty, Alexander (2012), “There's Something Queer Here”, Out in Culture: Gay, Lesbian and Queer Essays on Popular Culture, Corey K. Creekmur (ed.), Durham: Duke University Press, pp. 76-96.

Fone, Thomas (2019). "Persona y personaje en la escritura memorialística de Terenci Moix", Revista de Filología de la Universidad de La Laguna, N. ${ }^{\circ} 39$, pp. 185-200.

Huard, Geoffroy (2016), "Els homosexuals a Barcelona sota el franquisme. Prostitució, classe social i visibilitat entre 1956 i 1980", Franquisme Transició. Revista d'Història i de Cultu- 
ra, 2016, N. ${ }^{\circ}$ 4, pp. 127-51, URL: https://www.raco.cat/index.php/franquismeitransicio/ article/view/n4-huard

Kubicek, Katrina, Bryce McDavitt, Julie Carpineto, George Weiss, Ellen IverSOn, And Michele D. Kipke (2009), "'God Made Me Gay for a Reason’: Young Men Who Have Sex with Men's Resiliency in Resolving Internalized Homophobia from Religious Sources", Journal of Adolescent Research. Vol. 24, pp. 601-633. URL: https:// pubmed. ncbi.nlm.nih.gov/20160996/

MARI, Jorge (2000), "La astronomia de la pasion: espectadores y estrellas en El dia que murio Marilyn de Terenci Moix”, Modern Language Notes, Vol. 115, N. ${ }^{\circ}$ 2, pp. 224-247. URL: https://muse.jhu.edu/article/22615

Martel, Frédéric (2018), Global Gay: How Gay Culture Is Changing the World, Massachusetts: The MIT Press.

McGovern, Timothy (2004), "The Subversive Processes of Camp Production in the Novels of Terenci Moix", Journal of Iberian and Latin American Studies, Vol. 10, pp. 45-59. URL: https://doi.org/10.1080/1470184042000236279

Mira, Alberto (2007), De Sodoma a Chueca: Una historia cultural de la homosexualidad en Espana en el siglo $X X$, Barcelona: Egales.

Mira, Alberto (2016), "El niño queer en El peso de la paja de Terenci Moix", Masculinidades disidentes, Rafael M. Mérida Jiménez (ed.), Barcelona: Icaria, 2016, pp. 185-206.

Moix, Terenci (1998), El dia que murio Marilyn, Barcelona: Planeta.

Moix, Terenci (2002), Mujercísimas, Barcelona: Planeta.

Moix, Terenci (2013), Garras de astracán. Barcelona: Planeta.

Morales Guevara, Carmen (2008), "El espacio de la ciudad de Barcelona: Eduardo Mendoza y Terenci Moix”, Revista de Filología Románica, Vol. VI, pp. 169-175. URL: https://dx.doi. org/10.5209/RFRM

Olmeda, Fernando (2007), "La homosexualidad en España desde el Franquismo hasta hoy”, Cultura, homosexualidad y homofobia, vol. I: Perspectivas gays, Félix Rodriguez González (ed.), Barcelona: Laertes, pp. 21-32.

Ortega, María Luisa (2012), "De la españolada al fake. Estereotipos de la españolidad, identidad y diálogos transnacionales”, Nadia Lie (ed.), El juego con los estereotipos. La redefinición de la identidad hispánica en la literatura y el cine postnacionales, Bruselas: Peter Lang, pp. 99-118.

El PAís (1998), "Terenci Moix reescribe .El día que murió Marilynz "Mundo macho"”, Madrid: 16 diciembre de 1998. URL:https://elpais.com/diario/1998/12/16/cultura/913762804 850215.html

Sieburth, Stephanie (2014), Survival Songs: Conchita Piquer's Coplas and Franco's Regime of Terror, Toronto: University of Toronto Press.

Sontag, Susan (2018), Notes on 'camp', Londres: Penguin Modern.

Thomas, Harry (2013), "Ïmmaculate Manhood": The City and the Pillar, Giovanni's Room, and the Straight-Acting Gay Man”, Twentieth Century Literature, Vol. 59, No. 4, pp. 596-618. URL: https://doi.org/10.1215/0041462X-2013-1002

Vidal, Gore (2003), The City and the Pillar, New York: Vintage.

Recibido: junio de 2020

Aceptado: octubre de 2020 